\section{Recent trends and special topics in new drug review in PMDA}

\author{
Toshiki Sugita, PhD
}

The Pharmaceuticals and Medical Devices Agency (PMDA) is Japan's regulatory agency, which works together with the Ministry of Health, Labour and Welfare. At the 24th Drug Information Association Annual EuroMeeting in March 2012, PMDA presented an overview of recent trends and special topics in new drug review. Here, the current status and trends are highlighted, and the new drug review is discussed.

Keywords: Approval review, global clinical trials, Japan's regulatory agency, PMDA, prior assessment consultation

\section{Introduction}

At the 24th Drug Information Association Annual EuroMeeting in Copenhagen, Denmark, 26-28 March 2012, a presentation was made by the Pharmaceuticals and Medical Devices Agency (PMDA), Japan, titled 'Recent trends and special topics in new drug review in PMDA'. In this paper, I discussed key points of the new drug review. The views and opinions expressed here are my own, and should not be attributed to PMDA.

\section{New drug review and biosimilars approval guidelines in Japan}

PMDA evaluates the quality, safety, and efficacy of drugs in light of state-of-theart scientific and technological standards. In addition to the reviews produced by PMDA, other related services are provided. These include consultations on clinical trials in relation to regulatory submission; good laboratory practice; good clinical practice (GCP); good post-marketing surveillance practice inspections to ensure submitted data are in compliance with ethical and scientific standards; and good manufacturing practice and quality management system inspections to ensure quality management of the manufacturing facility for the product submitted for approval.

In the review of drug applications, PMDA reviewers form a team to evaluate the quality, pharmacology, pharmacokinetics, toxicology, clinical implications, and biostatistics relating to a particular drug product under review. The team comprises reviewers who have degrees in pharmaceutical science, medicine, veterinary medicine, physical science, biostatistics, or other specialties. During the review process, the reviewers exchange opinions with external experts (expert discussions) to ensure that effective reviews are conducted by making use of their advanced expertise. In addition, PMDA participates in the International Conference on Harmonisation of Technical Requirements for Registration of Pharmaceuticals for Human Use (ICH), and has actively incorporated the guidelines agreed upon at ICH into its drug reviews. It is the basic policy of a new drug review by PMDA.

The biosimilar review system in PMDA is almost the same as the new drug application as described above. The requirements for approval application of biosimilars are summarized in the 'Guideline for the quality, safety and efficacy assurance of follow-on biologics' [1]. It was issued by Japan's Ministry of Health, Labour and Welfare on 4 March 2009 [2]. Although there are a few differences between the requirements of Japanese guidelines and those of other countries such as the EU and the US, the fundamental concepts are similar [3]. Since the first approval of Sandoz's biosimilar somatropin in June 2009, one erythropoietin and two filgrastim biosimilars have been approved in Japan [2-4].

One of the greatest challenges of a new drug review by PMDA is the issue of 'drug lag' [2]. That is, drugs approved in the EU or the US that have not yet been approved in Japan and cannot be provided to Japanese citizens. Some reasons for drug lag in Japan include clinical trials starting later in Japan, and a longer time taken to conduct clinical trials and review new drugs.

The review time of new drugs has gradually reduced. In 2008, the median review time of new drugs (priority review) in Japan was 15.4 months. In 2010, however, it was 9.2 months, and in the first half of 2011, 6.8 months. The facilitation of median review time of new drugs (standard review) is similar to the priority review. In 2008, the time taken to review was 22.0 months. In 2010, however, it was 14.7 months and, in the first half of 2011 , it was 11.7 months. The total number of approved drugs has increased but review time has shortened over the years.

PMDA has made various efforts to resolve drug lag. To shorten the pre-application lag, the quality and quantity of consultations have improved, and global drug development has been promoted. In addition, PMDA has increased the number of reviewers, and enhanced and improved the review system. In particular, it is important to shorten the pre-application lag, because the pre-application lag is longer than the post-application lag. In the following section, the new category of consultations and the promotion of global drug development are discussed.

\section{Prior assessment consultation}

A prior assessment consultation is a new process introduced by PMDA's scientific consultation, which takes place before the submission of a formal new drug application (NDA). In a prior assessment consultation, PMDA reviews all data available, including chemistry, manufacturing and control, and non-clinical and clinical studies.

Prior assessment consultation may shorten NDA review time and maximize efficiency of the drug-development process by evaluating data before the formal submission of an NDA. Any critical issues for regulatory decision can therefore be resolved before proceeding to NDA review. 
Furthermore, PMDA can identify major discussion points and tasks, and the sponsor can prepare a well-organized, common technical document to meet PMDA's requirements before the NDA submission.

The time line of prior assessment consultations is about six months. Initially, the sponsor and PMDA discuss the consultation schedule, such as the date of document submission, inquiry, and final report. Then, the sponsor applies the consultation and submits the document, including chemistry and manufacturing control data, non-clinical, and clinical studies. PMDA evaluates the document and submits enquiries to sponsors within eight weeks from date of submission. The sponsor submits the response within six weeks from the date of enquiry submission. PMDA evaluates the document and sponsor's response, and prepares draft reports within seven weeks from the date of the sponsor's response. The sponsor checks the draft report. The sponsor and PMDA will discuss any comments to the draft report. The exchange of opinions is usually in writing. If necessary, however, face-to-face meetings will be held. Finally, PMDA will finalize the report and send it to the sponsor within three weeks from the date of drafting the report.

Prior assessment consultation began as a pilot project in April 2009. Sixteen products were selected in 2009 and 2010. PMDA surveyed sponsors at the end of the prior assessment consultation [5]. In response to the question 'Were consultation issues resolved or clarified?', $44 \%$ of sponsors answered 'resolved or clarified', $44 \%$ answered 'relatively clarified' and $12 \%$ answered 'neither'. This result shows that sponsors were generally satisfied with the prior assessment consultation.

In 2011, PMDA improved the prior assessment consultation. In the pilot project, prior assessment consultation was carried out once a year. Sponsors, however, hoped to increase the frequency of opportunities for consultation. In response to this request, the prior assessment consultation was increased to twice a year from 2011.

The next area for improvement is expansion of the assessment area covered. In the pilot project, prior assessment consultation covered phase I and phase II, but not phase III clinical trials data. Many sponsors felt that a new category, such as consultation of phase III data, was necessary. In response, PMDA created a new category: 'phase II/III consultation'. This new category covers clinical data assessment in later stages.

In prior assessment consultation, the sponsor and PMDA can share information and discussion points of new drugs before the NDA. Furthermore, PMDA can take time to discuss other points at NDA review. Furthermore, I think that submission of the entire data-package required for the NDA makes prior assessment consultation more beneficial for both sponsor and PMDA.

\section{Global clinical trials}

The number and percentage of global clinical trials are increasing annually in Japan. In 2007 , the number of global clinical trials was 38, accounting for about $7.5 \%$ of all clinical trials in Japan. In 2010, however, this rose to 130 global, an increase of over $20 \%$.

In Japan, a new guideline 'Global clinical trials: basic principles of global clinical trials' was published in 2007 [6]. This guideline shows the following basic requirements of global clinical trials:

- Clinical trials can be conducted in compliance with the ICH GCP.

- All participating countries and clinical sites can accept GCP audit from Japan.

- Along with consideration of factors that may affect efficacy and safety, subgroup analysis based on these factors can be possible to achieve.

- Social differences and practical situations can be properly understood.

This new guideline [6] encourages 'Japan's participation in global drug development' and promotes global clinical trials to consider ethnic factors more appropriately.

In reviewing global clinical trials, PMDA reviewers believe that information on efficacy and safety in Japanese participants is important. This is because, in some cases, different doses are approved for Japan compared with other regions. Furthermore, in some cases, dose-response relationships shown in bridging studies are different to that in corresponding studies.

Global clinical trials reviews can be complicated. For example, in large-scale global clinical trials, the percentage of Japanese participants is relatively low because the number of participating countries is large.
Therefore, low-incident events occur infrequently in Japanese participants. Thus, PMDA reviewers will always be particularly attentive to whether the results of global clinical trials can be regarded as evidence for the Japanese population.

In reviewing global clinical trials, intrinsic and extrinsic ethnic factors are checked, and results of Japanese participants are compared with non-Japanese participants. We check ethnic factors, in accordance with ICH-E5 [7]. With these comparisons, we check the results of efficacy and safety endpoints. We evaluate whether differences in ethnic factors are apparent between Japan and other regions, and whether the results show any similarities between Japanese participants compared with non-Japanese participants.

In addition to the primary endpoint, secondary or other endpoints are also important in supporting or evaluating ethnic similarities between Japan and other regions. As each individual drug undergoes a different development process, it has been suggested that PMDA consultation meetings are held as frequently as possible. Many development strategies are available, and it is important to discuss which strategy is the most appropriate for a particular drug.

\section{Competing interests: None.}

Provenance and peer review: Commissioned; externally peer reviewed.

\section{References}

1. Pharmaceuticals and Medical Devices Agency, Japan. MHLW, Guidelines for the quality, safety and efficacy assurance of follow-on biologics [homepage on the Internet]. [cited 2013 Apr 24]. Available from: http://www.pmda.go.jp/english/ service/pdf/notifications/PFSB-ELD-0304007. pdf

2. Ando Y, Tominaga T, Kondo T. PMDA update: the current situation and future directions. Generics and Biosimilars Initiative Journal (GaBI Journal). 2013;2(1):41-4. doi:10.5639/gabij.2013.0201.013

3. Yamaguchi T, Arato T. Quality, safety and efficacy of follow-on biologics in Japan. Biologicals. 2011;39(5):328-32

4. GaBI Online - Generics and Biosimilars Initiative. Japanese guidelines for biosimilars [www. gabionline.net]. Mol, Belgium: Pro Pharma Communications International; [cited 2013 Apr 24]. Available from: www.gabionline.net/Guidelines/ Japanese-guidelines-for-biosimilars

References 5-7 can be found on page 64 . 
Recent trends and special topics in new drug review in PMDA

References (please see the full manuscript on page 99-100)

5. Pharmaceuticals and Medical Devices Agency, Japan. Announcement of drug evaluation prior consultation survey overview [homepage on the Internet]. 2012 Mar 19 [cited 2013 Apr 24]. Japanese. Available from: http://www.pmda.go.jp/operations/shonin/info/consult/file/h21pas_survey.pdf
6. Pharmaceuticals and Medical Devices Agency, Japan. Basic principles on global clinical trials [homepage on the Internet]. 2007 [cited 2013 Apr 24]. Japanese. Available from: http:// www.pmda.go.jp/operations/notice/2007/ file/0928010-e.pdf
7. Pharmaceuticals and Medical Devices Agency, Japan. MHLW. ICH Harmonised tripartite guideline [homepage on the Internet]. 1998 [cited 2013 Apr 24]. Available from: http://www.pmda. go.jp/ich/e/e5r1_98_8_11e.pdf DOI: 10.5639/gabij.2013.0202.024

Copyright $\odot 2013$ Pro Pharma Communications International 